doi: 10.2306/scienceasia1513-1874.2014.40.340

\title{
RNAi down-regulation of Nck1 adaptor protein in Jurkat T cells
}

\author{
Pussadee Paensuwan $^{\mathrm{a}}$, Jatuporn Ngoenkam ${ }^{\mathrm{a}}$, Donruedee Sanguansermsri ${ }^{\mathrm{a}}$, Boonruang Khamsri ${ }^{\mathrm{a}}$, \\ Ichaya Yiemwattana $^{\mathrm{b}}$, Apirath Wangteeraprasert ${ }^{\mathrm{c}}$, Sutatip Pongcharoen ${ }^{\mathrm{c}, \mathrm{d}, *}$ \\ a Department of Microbiology and Parasitology, Faculty of Medical Science, Naresuan University, \\ Pitsanulok 65000 Thailand \\ b Department of Preventive Dentistry, Faculty of Dentistry, Naresuan University, Pitsanulok 65000 Thailand \\ ${ }^{c}$ Department of Medicine, Faculty of Medicine, Naresuan University, Phitsanulok 65000 Thailand \\ d Centre of Excellence in Medical Biotechnology, Faculty of Medical Science, Naresuan University, \\ Pitsanulok 65000 Thailand
}

*Corresponding author, e-mail: sutatipp@nu.ac.th, sutatipwp@yahoo.co.uk

\begin{abstract}
RNA interference (RNAi) is a potent gene delivery system for studying the regulation of gene expression in a wide variety of eukaryotic cells. In the present study, different RNAi approaches, namely, synthetic small interfering RNA (siRNA) and plasmid- and lentivirus-based short hairpin RNA (shRNA) were investigated to assess the down-regulation of Nck1 protein efficiency in the Jurkat $\mathrm{T}$ cell line. Jurkat $\mathrm{T}$ cells treated with these three different systems substantially and specifically reduced the expression of the Nck1 protein but not that of the Nck2 protein. Although the three systems showed a similar Nck1 knockdown efficiency, they led to different T cell activation outcomes. After stimulation, CD69 expression and IL-2 production were impaired in Nck1-siRNA and plasmid-based Nck1-shRNA transfected Jurkat cells. However, these $T$ cell activation outcomes were increased in lentiviral vector based Nck1-shRNA transfected cells. These data suggest that the outcomes from transfection with the shRNA based lentiviral vector differ from those of siRNA and shRNA-based plasmids although they provide the same gene silencing efficiency. The verification of suitable RNAi strategies to silence target genes is therefore necessary before using it in experiments.
\end{abstract}

KEYWORDS: RNA interference, lentiviral vector, gene silencing

\section{INTRODUCTION}

RNA interference (RNAi) is a powerful technique to investigate mammalian gene function by sequencespecific gene silencing. RNAi, first discovered in the nematode Caenorhabditis elegans ${ }^{1}$, is the biological process underlying double-stranded RNA (dsRNA)induced gene silencing resulting in the inhibition of specific gene expression. The RNAi hallmark is the specificity of the dsRNA in which sequences are homologous to that of the target $\mathrm{mRNA}^{2}$. Experimentally, RNAi can be achieved by transfecting cells either directly using synthetic siRNA constructs or by using DNA vectors or viral vectors encoding short hairpin (sh) $\mathrm{RNA}^{3}$.

Small interfering RNAs (siRNAs) comprise of specific sequences of 19-21 nucleotides that can be chemically synthesized consisting of sense and complementary anti-sense strands. These complementary strands are annealed to form a double stranded siRNA duplex, which is then introduced into host cells by various transfection techniques. siRNAs can reduce the expression of the gene of interest by enzymatic degradation of a target mRNA via the RNA-induced silencing complex. The efficiency of siRNA is that it specifically reduces the expression of the gene of interest without influencing irrelevant genes. However, the synthetic siRNA provides a transient knockdown of the target gene as it undergoes enzymatic degradation in the host cells or becomes diluted in rapidly dividing cells ${ }^{4}$. The effectiveness of gene knockdown can be prolonged using vectorbased approaches where a shRNA expression system continually generates siRNA-like species ${ }^{5}$. The shRNA consists of a siRNA sequence followed by a 9 nucleotide loop and a reverse complementary siRNA sequence, which is then inserted into a plasmid or viral vector. In comparison to the plasmid-based shRNA system, some viral-based shRNA types, such as the lentiviral vector, can integrate their shRNA-encoding genome stably into a host genome resulting in stable gene knockdown. The lentivirus is commonly used for 
long term gene silencing because it can be transfected into a wide variety of cell lines and primary cells both in in vitro and in vivo ${ }^{3,5,6}$.

The noncatalytic region of tyrosine kinase (Nck) is an adaptor protein expressed in a variety of tissue cells and cell lines. The Nck family consists of two known members in humans, including Nck $1 /$ Nck $\alpha$ and $\mathrm{Nck} 2 / \mathrm{Nck} \beta$. Each Nck isoform contains three src homology 3 (SH3) domains and one $\mathrm{SH} 2$ domain. In many cell types, Nck links phosphotyrosine signals and regulates actin cytoskeletal rearrangements ${ }^{7}$. In T cells activated via their $\mathrm{T}$ cell antigen receptor (TCR), Nck is essential in regulating actin rearrangement and immunological synapse formation ${ }^{8-14}$. Interestingly, our recent data have shown that silencing of Nck1 by transfection of siRNA to Jurkat T cell results in abrogation of $\mathrm{T}$ cell activation as indicated by decreased interleukin-2 (IL-2) secretion and CD69 expression ${ }^{14}$.

RNAi is commonly achieved by either siRNA, plasmid-based shRNA or viral-based shRNA; however, researchers normally rely on one of these methods. Our research therefore examines whether these three RNAi systems would be equally effective in gene knockdown. For this, two important readouts associated with $\mathrm{T}$ cell activation were examined: CD69 expression and IL-2 production.

\section{MATERIALS AND METHODS}

\section{Cell culture}

Jurkat E6.1 T cells (clone E6-1; American Type Culture Collection, Rockville, MD, USA) were cultured in RPMI-1640 medium (Gibco, Gaithersburg, MD, USA) supplemented with $10 \%$ foetal bovine serum (Gibco) and 1\% penicillin/streptomycin (JRH Biosciences, Victoria, Australia), and $2 \mathrm{mM}$ L-glutamine (JRH Biosciences). Cells were grown in a humidified incubator at $37^{\circ} \mathrm{C}$ with $5 \% \mathrm{CO}_{2}$.

\section{Nck siRNA transfection}

The siRNA duplex sequences targeting Nck1 and control siRNA were designed and synthesized chemically by Invitrogen (Invitrogen, Carlsbad, CA, USA). The siRNA sequence targeting Nck1 used in this study was 5'-GGGTTCTCTGTCAGAGAAA-3'. The siRNA transfection was performed as previously described ${ }^{14}$. Briefly, $2 \times 10^{5}$ Jurkat cells were harvested, washed with D-PBS (Invitrogen) and resuspended in $10 \mu \mathrm{l}$ solution R plus 50 pmol of stealth RNAi siRNA duplexes specific for Nck1. Negative controls were performed by using negative stealth siRNA low in GC content (Invitrogen). Cells were then electroporated in a microporator pipette by MicroPorator (Digital Bio
Technology, Seoul, Korea) using three pulses of $10 \mathrm{~ms}$ at $1600 \mathrm{~V}$. The transfected cells were used after $48 \mathrm{~h}$ post transfection.

\section{Nck1-shRNA plasmid construction and transfection}

The Nck1-shRNA construct was designed according to the Lenti-X Lentiviral Expression Systems manual (Clontech, Mountain View, CA). The Nck1-shRNA sequence was 5'-GATCCGGGGTTCTCTGTCAG AGAAATTCAAGAGATTTCTCTGACAGAGAA CCCTTTTTTACGCGTG-3'. The sense sequence targeting Nck1 was designed based on the Nck1siRNA oligonucleotide. The Nck1-shRNA was 64 nucleotides in length consisting of 19 nucleotide sense and antisense sequences separated by a 9 nucleotide noncomplementary loop sequence. This Nck1shRNA oligonucleotide was flanked with a BamHI restriction site at the $5^{\prime}$ end and an RNA polymerase III termination signal followed by an EcoRI restriction site at the $3^{\prime}$ end. The Nck1-shRNA oligonucleotides were cloned into the pLVX-shRNA1 vector (Clontech) at the multiple cloning sites downstream of the U6 promoter. The constructed plasmid was transformed into competent cells and the positive clones were subsequently confirmed by sequencing. Transfection of constructed plasmid into Jurkat T cells was carried out as described above. Briefly, $2 \times 10^{5}$ Jurkat T cells were transfected with either $1 \mu \mathrm{g}$ Nck1-shRNA or control scramble expressing plasmid (Addgene). After incubation for $48 \mathrm{~h}, 4 \mu \mathrm{g} / \mathrm{ml}$ of puromycin was added into the culture to generate stable Nck1knockdown cell lines.

\section{Lentiviral particle production and transduction}

Lentiviral supernatant was generated in HEK293T cells. Briefly, the $293 \mathrm{~T}$ cells were cultured in the DMEM medium supplemented with $10 \%$ tetracyclinefree FBS (Clontech) and $2 \mathrm{mM}$ L-glutamine. Approximately $24 \mathrm{~h}$ before transfection, $1 \times 10^{7}$ cells were plated on a $100 \mathrm{~mm}$ culture dish containing $10 \mathrm{ml}$ of complete medium and incubated at $37^{\circ} \mathrm{C}$, $5 \% \mathrm{CO}_{2}$ overnight. To produce Nck1-specific shRNA lentivirus, cells were co-transfected with $1 \mu \mathrm{g} / \mu \mathrm{l}$ of the constructed Nck1-shRNA plasmid and Lenti-X HTX packaging mix (Clontech) using Xfect kit (Clontech). shRNA control (scramble shRNA) lentivirus supernatant was prepared by co-transfection of HEK293T cells with $4 \mu \mathrm{g}$ of pCMV-dR8.2 dvpr, $4 \mu \mathrm{g}$ of pCMVVSV-G, and $7 \mu \mathrm{g}$ scramble shRNA vector (Addgene). Four hours post-transfection, the cultures were refreshed with $10 \mathrm{ml}$ fresh medium and incubated at $37^{\circ} \mathrm{C}$ for an additional $24-48 \mathrm{~h}$. Viral super- 
natants were concentrated using Lenti-X Concentrator according to manufacturer's instructions (Clontech). Lentiviral titter was determined using a Lenti-X qRTPCR titration kit (Clontech).

For transduction of lentivirus to target cells, $2 \times 10^{5}$ Jurkat $\mathrm{T}$ cells were cultured in complete medium for $12-18 \mathrm{~h}$ before transduction. Jurkat $\mathrm{T}$ cells were then transduced with the lentiviral particles with multiplicity of infection 10 and in the presence of $4 \mu \mathrm{g} / \mathrm{ml}$ of polybrene. After $24 \mathrm{~h}$ of incubation, the cultured medium was replaced with fresh medium and further incubated for 24-48 h followed by incubating cells in medium containing $4 \mu \mathrm{g} / \mathrm{ml}$ puromycin for 4 7 days.

\section{Immunoblotting}

Protein samples were resolved on $10 \%$ sodium dodecyl sulphate-polyacrylamide gel and transferred to a polyvinylidene difluoride (PVDF) membrane (Pall, Ann Arbor, MI, USA). The membrane was blocked with RapidBlock solution (Amresco, Solon, Ohio, USA). The membrane was then incubated with rabbit monoclonal antibodies against human Nck1 (Cell Signalling Technology, Danver, MA, USA), or Nck2 (Abnova Corp., Taipei, Taiwan) or $\beta$-actin (Cell Signalling Technology). The membrane was developed using enhanced chemiluminescence reagent (ECL: Bio-Rad, USA) and was then observed by the ImageQuant LAS 4000 imaging system (GE healthcare, Uppsala, Sweden).

\section{IL-2 production and measurement}

Jurkat E6.1 T cells in the logarithmic growth phase were harvested and resuspended in complete medium. $2 \times 10^{5}$ cells were then seeded into a 96-well tissue culture plate pre-coated with $1 \mu \mathrm{g} / \mathrm{ml}$ anti-CD3 antibody (OKT3, eBioscience, San Diego, CA, USA) in the presence of $1 \mu \mathrm{g} / \mathrm{ml}$ soluble anti-CD28 antibody (eBioscience, San Diego, CA, USA). Cells were then incubated for $24 \mathrm{~h}$ at $37^{\circ} \mathrm{C}$ with $5 \%$ $\mathrm{CO}_{2}$. Secreted IL-2 in the supernatant was determined using a commercial enzyme-linked immunosorbent assay (ELISA) kit (R\&D, Minneapolis, MN, USA) following the manufacturer's instructions. The optical density at $450 \mathrm{~nm}$ was read using a microplate reader (Perkin Elmer).

\section{Detection of CD69 expression by flow cytometry}

The expression of CD3 molecules on $\mathrm{T}$ cell surface was determined using anti-CD3 conjugated FITC antibody. To examine the expression of CD69, Jurkat T cells were stimulated as described above. Cells were washed twice with PBS containing $1 \%$ FBS, and then

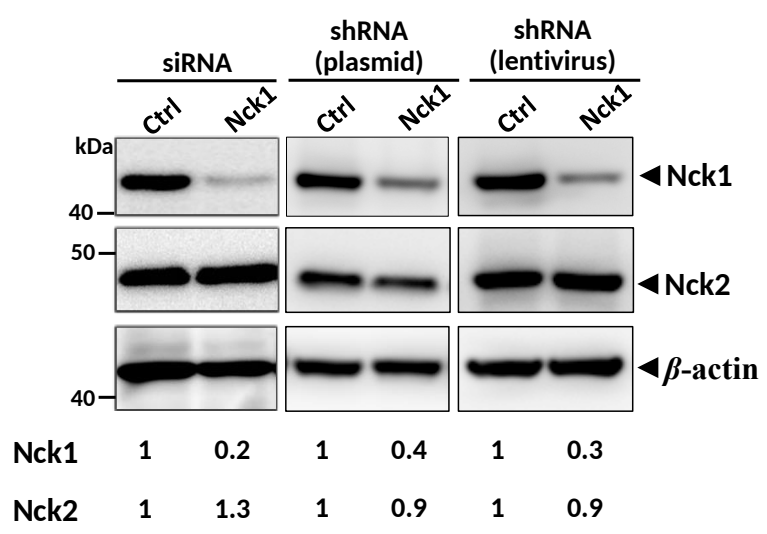

Fig. 1 Nck1 protein expression was reduced in siRNA, plasmid- and lentiviral-based shRNA systems. Jurkat T cells were treated with Nck1-specific siRNA (siRNA), or plasmid encoding Nck1-specific shRNA (plasmid), or lentivirus generating Nck1-specific shRNA (lentivirus). Protein expression was assessed by immunoblotting using anti-Nck1, Nck 2 and $\beta$-actin antibody and was quantified by the ImageJ software. The quantified signal intensities of the Nck1 or Nck2 were normalized to their corresponding actin and these values were relative to that in the control cells $(\mathrm{Ctl})$, set as 1 . Numbers below the band indicate expression level of Nck1 and Nck2 compared with control (Ctl). One representative experiment out of two is shown.

incubated with phycoerythrin-conjugated mouse antihuman CD69 mAb or isotype control for $30 \mathrm{~min}$ at $4{ }^{\circ} \mathrm{C}$ in the dark. Finally, cells were assessed for CD69 expression using an FACScalibur (Becton Dickinson, NJ, USA) and analysed with CELLQUESTPRO.

\section{RESULTS}

\section{All three RNAi strategies provided an effective down-regulation of the Nck1 protein in Jurkat $T$ cells}

The ability of the RNAi system to silence gene expression has been proven to be invaluable for studying gene function in cultured mammalian cells. The achievement or failure to silence a gene of interest depends on RNAi delivery systems and cell types. In this study, Jurkat E6.1 T cells were transfected with either Nck1-siRNAs or plasmid-based Nck1shRNAs or transduced with lentiviral-based Nck1shRNA. These three approaches showed a reduction of Nck1 protein expression level to about $75 \%$ (Fig. 1). In addition, these three techniques exhibited gene-specific silencing since they did not affect the expression of a related protein such as Nck2. Thus both non-viral and viral vector methods successfully delivered Nck1siRNA and Nck1-shRNA into target 

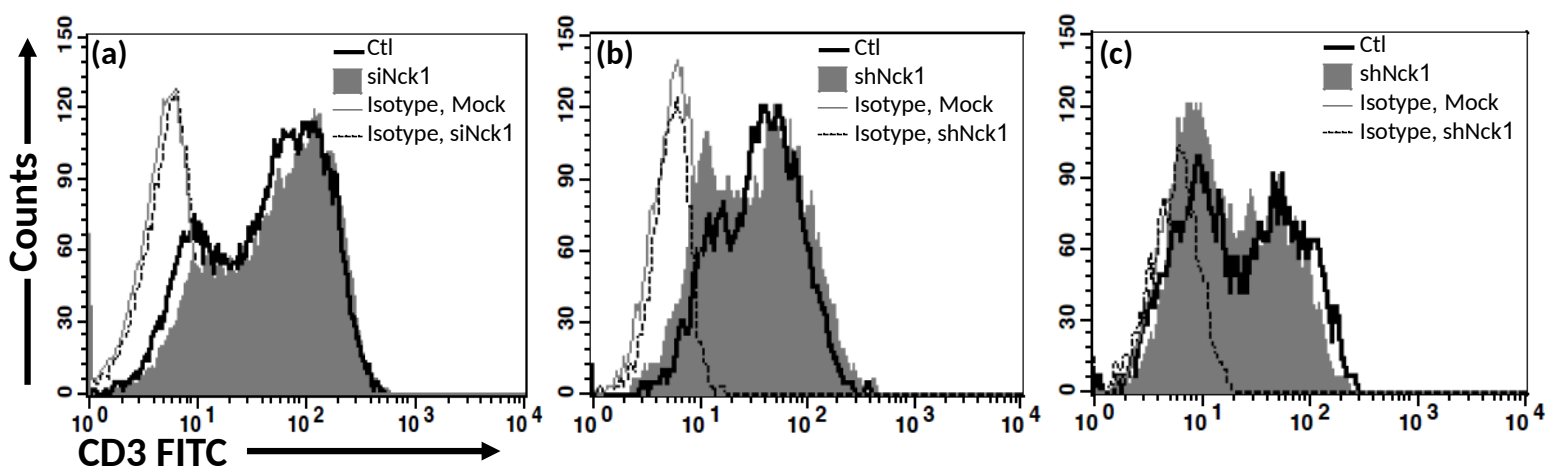

Fig. 2 TCR expression level in Jurkat T cells. Jurkat T cells were treated with (a) Nck1-siRNA, (b) shNck1 plasmids, and (c) shNck1 lentivirus. The TCR expression of control cells (Ctl) (bold solid line) and Nck1-knockdown cells (siNck1 or shNck1) (grey histogram) was determined by staining cells with anti-CD3 conjugated FITC antibody or isotype control antibodies. The stained cells were then analysed by flow cytometer. The dotted line represents the isotype control in each group. The data are representative of two independent experiments.

cells to mediate specific gene silencing.

\section{Three RNAi systems did not affect TCR expression}

The $\mathrm{T}$ cell receptor (TCR) together with its associated CD3 signalling subunits (TCR-CD3 complex) is essential for antigen recognition and activation of $\mathrm{T}$ cell. The effect of these RNAi systems on TCR expression was monitored. The TCR-CD3 complex molecules on the cell surface were stained with anti-CD3 conjugated FITC antibody and analysed by flow cytometry. The results indicated that there were no significant differences of the TCR expression level among these RNAi systems (Fig. 2). Hence the silencing of Nck1 expression by these three different techniques did not impair TCR expression on the T cell surface.

\section{CD69 expression and IL-2 production depended on RNAi-mediated Nck1 silencing techniques}

$\mathrm{T}$ cell activation normally leads to the expression of the CD69 molecule and production of numerous cytokines and chemokines, which are important for the immune response. CD69 is a type-II C-type lectin receptor, known as one of the earliest cell surface activation markers ${ }^{15}$. The upregulation of CD69 surface expression on $\mathrm{T}$ cells is followed by cell proliferation and an increase in the secretion of IL-2 and IFN- $\gamma$. In this study, CD69 expression and $\mathrm{IL}-2$ production were examined and compared between cells treated with different RNAi-mediated Nck1 silencing techniques. Nck1-knockdown cells obtained from three RNAi approaches were stimulated with $1 \mu \mathrm{g} / \mathrm{ml}$ anti-CD3 OKT3 antibody plus $1 \mu \mathrm{g} / \mathrm{ml}$ anti-CD28 antibody for $24 \mathrm{~h}$. Cells were then analysed for CD69 expression by flow cytometry and supernatants containing IL-2 were quantitatively measured by ELISA. It was found that CD69 expression was impaired in Nck1 knockdown cells using siRNA and plasmid-based shRNA treatment, but not Nck1 knockdown cells using lentiviral-based shRNA treatment (Fig. 3). The results were consistent with IL-2 production (Fig. 4).

\section{DISCUSSION}

RNAi-based gene silencing is commonly used to study gene function in $\mathrm{T}$ cell responses ${ }^{4,16-19}$. Here we compared three different RNAi strategies, including synthetic siRNA, plasmid- and lentivirusmediated shRNA production in silencing of the Nck1 gene and their outcomes after cell activation. Among these three different RNAi methods, synthetic doublestranded siRNA with about 21 nucleotides is more potent in mediating sequence-specific gene silencing as it resembles natural cellular machinery. However, introducing double-stranded siRNA molecules of over 50 nucleotides long can induce an interferon response in mammalian cells, resulting in translation inhibition and mRNA degradation of non-specific gene ${ }^{20,21}$. In this study, Nck1-specific siRNA duplexes of 19 nucleotides long were used to transfect into Jurkat $\mathrm{T}$ cells for suppressing Nck1 expression. Although synthetic siRNA duplexes can be used to avoid non-specific global suppression of gene expression caused by the interferon responses ${ }^{2}$, the transient nature in gene silencing of siRNA makes it unsuitable for generation of stable gene knockdown in Jurkat cell lines. Hence plasmid- and lentiviral-based shRNA systems have been used to achieve prolonged gene silencing as they 

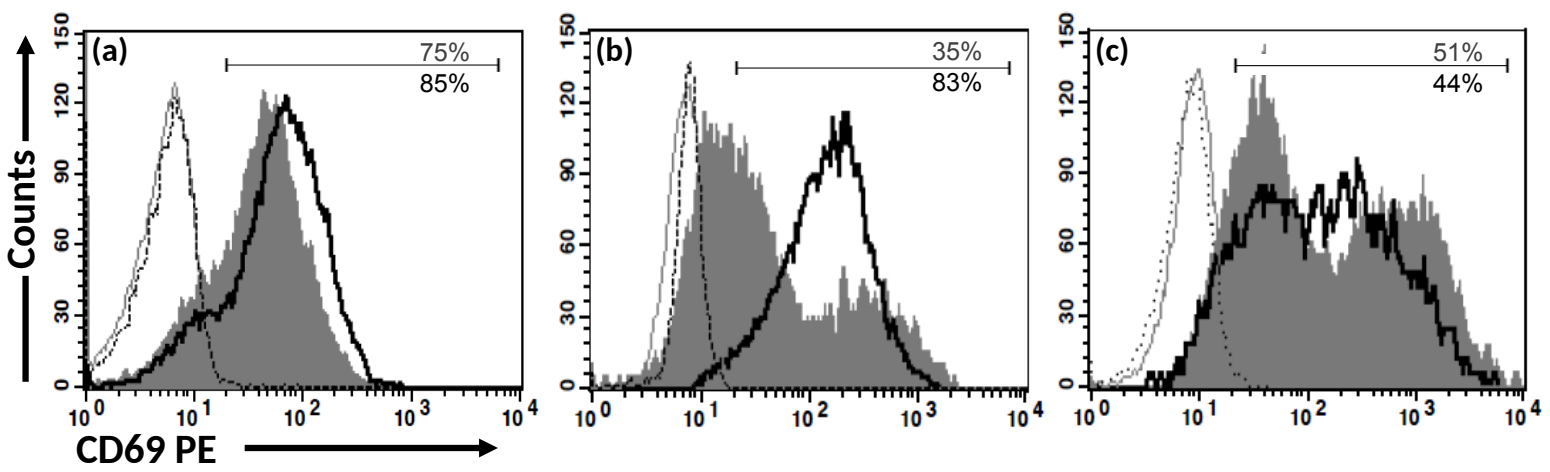

Fig. 3 CD69 expression was down-regulated in Nck1-siRNA and plasmid-based Nck1-shRNA but was up-regulated in lentivirus-based Nck1-shRNA systems. Nck1 knockdown cells mediated by different systems including (a) Nck1-siRNA, (b) plasmid- based Nck1shRNA, and (c) lentiviral-based Nck1 shRNA, were stimulated with anti-CD3 antibody precoated on the plate and soluble anti-CD28 antibody for $24 \mathrm{~h}$ at $37^{\circ} \mathrm{C}$. CD69 expression was assessed by flow cytometer. Numbers in CD69 histogram indicate frequency of positive cells. Black bold solid line and black letter are control cells, grey histogram and grey letter are Nck1 knockdown cells, and dotted and grey lines are isotype control. The data are representative of two independent experiments.
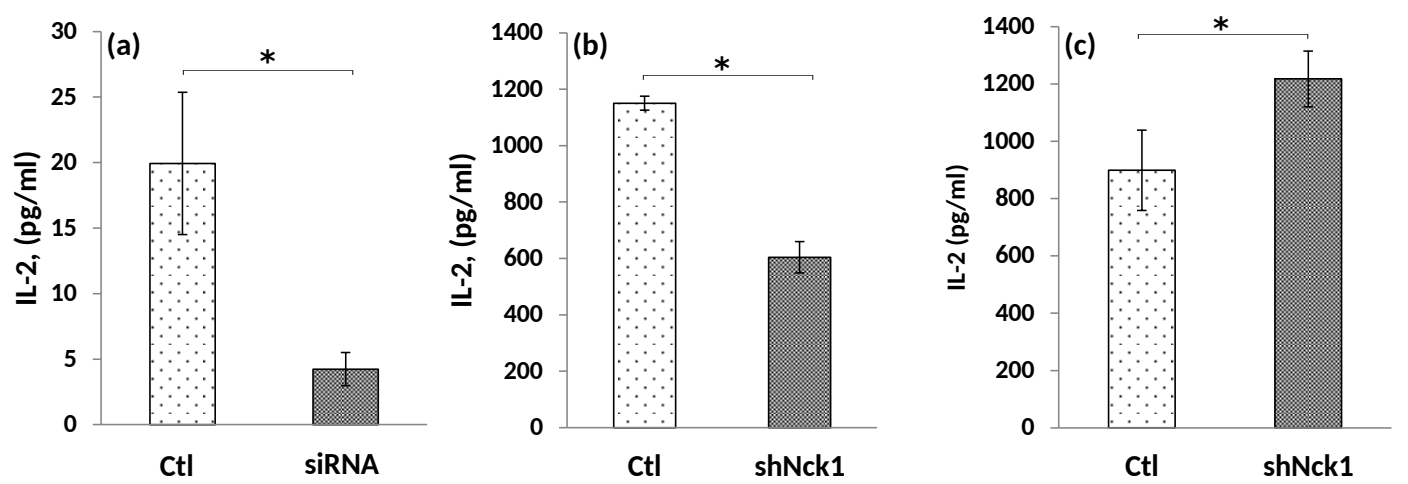

Fig. 4 The production of IL-2 was impaired in Nck1-siRNA and plasmid-based Nck1-shRNA transfected Jurkat cells but was upregulated in lentivirus-based Nck1-shRNA transduced Jurkat cells. Nck1 knockdown cells mediated by different systems including (a) Nck1-siRNA, (b) plasmid- based Nck1shRNA, and (c) lentiviral-based Nck1 shRNA, were stimulated with anti-CD3 antibody precoated on the plate and soluble anti-CD28 antibody for $24 \mathrm{~h}$ at $37^{\circ} \mathrm{C}$. Supernatants containing IL-2 were measured by ELISA. Data are representative of two independent experiments and shown as mean $\pm \mathrm{SD}$ from triplicate samples. ${ }^{*} p<0.05$.

endogenously and continuously express shRNAs in Jurkat $\mathrm{T}$ cells. These shRNAs are then processed in the cytoplasm to siRNA duplexes by a cellular enzyme called endoribonuclease dicer ${ }^{22}$.

For shRNA expression systems, the proper design of shRNA expression cassettes is required. The stable expression of shRNA is driven by RNA polymerase III promoters, $\mathrm{H} 1$, or U6 promoters ${ }^{23,24}$. In contrast to the H1 promoter, the U6 promoter leads to a high level of shRNA expression, which provides a stronger reduction of the target gene ${ }^{25}$. However, it has been found that a flawed vector design of the U6shRNA vector can cause the IFN response ${ }^{26}$. This IFN response is induced by the presence of $\mathrm{AA}$ residues at the transcription start site $(-1 /+1)$ of the U6-shRNA vector. Replacement of $\mathrm{AA}$ with a $\mathrm{C} / \mathrm{G}$ sequence at position $-1 /+1$ could prevent IFN induction ${ }^{26}$. In the present study, we used the U6-shRNA vector (pLVX) to construct shRNA expression cassettes. This vector contains a consensus $\mathrm{C} / \mathrm{G}$ at position $-1 /+1$ of the transcription start site. These constructed plasmids can be directly transfected into Jurkat $\mathrm{T}$ cells for endogenous expression of shRNA specific to the Nck1 gene. Alternatively, they can be combined with lentiviral packaging plasmids to produce lentiviruses, which can infect Jurkat cells and continually expresses shRNA. The use of lentiviruses to mediate stable gene silencing is more powerful since they can be applied 
Table 1 Summary of advantages and disadvantages of different transfection techniques.

\begin{tabular}{lll}
\hline Transfection technique & Advantages & Disadvantages \\
\hline Electroporation & $\begin{array}{l}\text { Nonchemical method } \\
\text { Does not alter the biological structure or } \\
\text { functions of target cells } \\
\text { Easy to perform } \\
\text { High efficiency }\end{array}$ & $\begin{array}{l}\text { Loss of cell viability } \\
\text { Requires parameter optimization for dif- } \\
\text { ferent cell types }\end{array}$ \\
& Able to apply to a wide range of cell types & \\
Lentiviral delivery system & $\begin{array}{l}\text { High gene delivery efficiency } \\
\text { Simplicity of infection } \\
\end{array}$ & $\begin{array}{l}\text { Labour intensive } \\
\text { Able to transduce to a wide range of cell } \\
\text { types, including non-dividing and dividing } \\
\text { mammalian cells }\end{array}$ \\
& Stable gene expression & Requires special bio-safety equipment \\
& & \\
\hline
\end{tabular}

in dividing and non-dividing cells as well as cells that are difficult to transfect.

In humans, Nck1 shares $68 \%$ amino acid identity with $\mathrm{Nck} 2^{7}$. Jurkat $\mathrm{T}$ cells treated by these three different systems achieved similar reduction of Nck1 expression by about $60-80 \%$ without affecting the expression of Nck2. This indicates sequence-specific gene silencing of these systems. Notably, although these three different RNAi strategies were effectively able to knockdown the Nck1 gene in Jurkat T cells, they showed distinct phenotypes as observed by CD69 expression and IL-2 production. These readouts are the primary indicators of $\mathrm{T}$ cell activation. Here, we found that knockdown of Nck1 by siRNA and shRNA expression plasmids impair CD69 expression and IL-2 production during TCR-mediated Jurkat T cell activation. Surface expression of CD69 is regulated through the Ras activation pathway, which is essential for IL-2 gene transcription and production ${ }^{27}$. Moreover, Nck has been known to interact with son of sevenless (SOS) factor, which is required to control the activation of $\operatorname{Ras}^{28-30}$. Thus the reduced CD69 expression and IL-2 secretion were probably due to the loss of Nck that interact with SOS. In agreement with this notion, mutation on the third $\mathrm{SH} 3$ domain of Nck1 to inhibit Nck-SOS interaction has been found to abrogate CD69 expression and Erk activation ${ }^{31}$. In sharp contrast, expression of CD69 and production of IL-2 were up-regulated in Jurkat cells transduced with lentiviruses to mediate Nck1 knockdown. The random integration of lentiviral DNA into the host cell's genome may result in the insertional mutagenesis of an unpredictable gene ${ }^{32,33}$, which may cause the upregulation of CD69 expression and IL-2 production. Altogether, we show that these three RNAi methods provide inconsistent results.
Although several pieces of evidence have shown that transfection of siRNA or transduction of lentivirus to express shRNA could activate the interferon signalling and interferon-stimulated gene ${ }^{26,34,35}$, RNAi technology is still the great promise for gene function analysis in $\mathrm{T}$ cell research. All three methods had advantages and disadvantages depending on research applications and facilities available in the laboratory, as summarized in Table 1. Identification of suitable RNAi techniques as well as designing correct shRNA expression cassettes and systems are therefore essential before proceeding with the experiments. Furthermore, functional outcomes should be examined empirically.

In conclusion, this study provides comparison of three RNAi delivery methods to the Jurkat E6.1 T cell line. This included the siRNA, vector-based shRNA transfection, and shRNA lentiviral vector transduction. We found that siRNA and vector-based shRNA transfection provided similar results while these differed from shRNA lentiviral vector system.

Acknowledgements: Pussadee Paensuwan and Jatuporn Ngoenkam are supported by the Royal Golden Jubilee PhD programme of the Thailand Research Fund. This work was supported by Naresuan University Research Grants No. R2556B026, R2557B029 (to SP) and R2557B024 (to IY). The authors would like to thank Kwansuda Supalap, Pimporn Udompol, and Sang Sri-ampai, Biomedical Research Unit, Office of Research, Faculty of Medicine, Naresuan University, for technical assistance. The authors also thank Dr Sarawut Kumphune and Dr Rerngwit Boonyom, Faculty of Allied Health Sciences, as well as Dr Pannika Ritvirool, Department of Microbiology and Parasitology, Faculty of Medical Science, Naresuan University for technical advice. 


\section{REFERENCES}

1. Fire A, Xu S, Montgomery MK, Kostas SA, Driver SE, Mello CC (1998) Potent and specific genetic interference by double-stranded, RNA in Caenorhabditis elegans. Nature 19, 806-11.

2. Elbashir SM, Harborth J, Lendeckel W, Yalcin A, Weber K, Tuschl T (2001) Duplexes of 21-nucleotide RNAs mediate RNA interference in cultured mammalian cells. Nature 411, 494-8.

3. Li F, Mahato RI (2011) RNA interference for improving the outcome of islet transplantation. Adv Drug Deliv Rev 63, 47-68.

4. Manjunath N, Wu H, Subramanya S, Shankar P (2009) Lentiviral delivery of short hairpin RNAs. Adv Drug Deliv Rev 25, 732-45.

5. Miest T, Saenz D, Meehan A, Llano M, Poeschla EM (2009) Intensive RNAi with lentiviral vectors in mammalian cells. Methods 47, 298-303.

6. Tiscornia G, Singer O, Ikawa M, Verma IM (2003) A general method for gene knockdown in mice by using lentiviral vectors expressing small interfering RNA. Proc Natl Acad Sci USA 18, 1844-8.

7. Lettau M, Pieper J, Janssen O (2009) Nck adaptor proteins: functional versatility in T cells. Cell Comm Signal 7, 1.

8. Gil D, Schamel WWA, Montoya M, Sánchez-Madrid F, Alarcón B (2002) Recruitment of Nck by CD3 $\varepsilon$ reveals a ligand-induced conformational change essential for $\mathrm{T}$ cell receptor signaling and synapse formation. Cell 109, 901-12.

9. Barda-Saad M, Shirasu N, Pauker MH (2010) Cooperative interactions at the SLP-76 complex are critical for actin polymerization. EMBO J 29, 2315-28.

10. Barda-Saad M, Braiman A, Titerence R, Bunnell SC, Barr VA, Samelson LE (2005) Dynamic molecular interactions linking the $\mathrm{T}$ cell antigen receptor to the actin cytoskeleton. Nat Immunol 6, 80-9.

11. Li W, Fan J, Woodley DT (2001) Nck/Dock: an adapter between cell surface receptors and the actin cytoskeleton. Oncogene 20, 6403-17.

12. Tailor P, Tsai S, Shameli A, Serra P, Wang J, Robbins S, Nagata M, Szymczak-Workman AL, Vignali DA, Santamaria $\mathrm{P}(2008)$ The proline-rich sequence of $\mathrm{CD} 3 \mathcal{\varepsilon}$ as an amplifier of low-avidity TCR signaling. J Immunol 181, 243-55.

13. Risueño RM, Gil D, Fernández E, Sánchez-Madrid F, Alarcón B (2005) Ligand-induced conformational change in the T-cell receptor associated with productive immune synapses. Blood 106, 601-8.

14. Yiemwattana I, Ngoenkam J, Paensuwan P, Kriangkrai R, Chuenjitkuntaworn B, Pongcharoen S (2012) Essential role of the adaptor protein Nck1 in Jurkat T cell activation and function. Clin Exp Immunol 167, 99-107.

15. Marzio R, Jirillo E, Ransijn A, Mauël J, Corradin SB (1997) Expression and function of the early activation antigen CD69 in murine macrophages. J Leukoc Biol 62, 349-55.

16. Pauker MH, Reicher B, Fried S, Perl O, Barda-Saad M (2011) Functional cooperation between the proteins Nck and ADAP is fundamental for actin reorganization. Mol Cell Biol 31, 2653-66.

17. Warnecke N, Poltorak M, Kowtharapu BS, Arndt B, Stone JC, Schraven B, Simeoni L (2012) TCR-mediated Erk activation does not depend on Sos and Grb2 in peripheral human T cells. EMBO Rep 13, 386-91.

18. Green VA, Arbuthnot P, Weinberg MS (2012) Impact of sustained RNAi-mediated suppression of cellular cofactor Tat-SF1 on HIV-1 replication in CD4+ T cells. Virol J 9, 272

19. Sylvain NR, Nguyen K, Bunnell SC (2011) Vav1mediated scaffolding interactions stabilize SLP-76 microclusters and contribute to antigen-dependent $\mathrm{T}$ cell responses. Sci Signal 4, ra14.

20. Stark GR, Kerr IM, Williams BR, Silverman RH, Schreiber RD (1998) How cells respond to interferons. Annu Rev Biochem 67, 227-64.

21. Bernstein E, Caudy AA, Hammond SM, Hannon GJ (2001) Role for a bidentate ribonuclease in the initiation step of RNA interference. Nature 409, 363-6.

22. Abbas-Terki T, Blanco-Bose W, Déglon N, Pralong W, Aebischer P (2002) Lentiviral-mediated RNA interference. Hum Gene Ther 13, 2197-201.

23. Banerjea A, Li MJ, Bauer G, Remling L, Lee NS, Rossi J, Akkina R (2003) Inhibition of HIV-1 by lentiviral vector-transduced siRNAs in T lymphocytes differentiated in SCID-hu mice and CD34+ progenitor cell-derived macrophages. Mol Ther 8, 62-71.

24. Fish RJ, Kruithof EK (2004) Short-term cytotoxic effects and long-term instability of RNAi delivered using lentiviral vectors. BMC Mol Biol 5, 9.

25. Robbins MA, Li M, Leung I, Li H, Boyer DV, Song Y, Behlke MA, Rossi JJ (2006) Stable expression of shRNAs in human CD34+ progenitor cells can avoid induction of interferon responses to siRNAs in vitro. Nat Biotechnol 24, 566-71.

26. Sledz CA, Holko M, de Veer MJ, Silverman RH, Williams BR (2003) Activation of the interferon system by short-interfering RNAs. Nat Cell Biol 5, 834-9.

27. Finco TS, Kadlecek T, Zhang W, Samelson LE, Weiss A (1998) LAT is required for TCR-mediated activation of PLC $\gamma 1$ and the Ras pathway. Immunity 9, 617-26.

28. Wunderlich L, Farago A, Buday L (1999) Characterization of interactions of Nck with Sos and dynamin. Cell Signal 11, 25-9.

29. Hu Q, Milfay D, Williams LT (1995) Binding of NCK to SOS and activation of ras-dependent gene expression. Mol Cell Biol 15, 1169-74.

30. Okada S, Pessin JE (1996) Interactions between Src homology (SH) 2/SH3 adapter proteins and the guanylnucleotide exchange factor SOS are differentially regulated by insulin and epidermal growth factor. $J$ Biol Chem 271, 25533-8. 
31. Ngoenkam J, Paensuwan P, Preechanukul K, Khamsri B, Yiemwattana I, Beck-García E, et al (2014) Nonoverlapping functions of Nck1 and Nck2 adaptor proteins in T cell activation. Cell Comm Signal 12, 21.

32. Li Z, Kustikova OS, Kamino K, Neumann T, Rhein M, Grassman E, Fehse B, Baum C (2007) Insertional mutagenesis by replication-deficient retroviral vectors encoding the large T oncogene. Ann New York Acad Sci 1106, 95-113.

33. Baum C, Fehse B (2003) Mutagenesis by retroviral transgene insertion: risk assessment and potential alternatives. Curr Opin Mol Therapeut 5, 458-62.

34. Bridge AJ, Pebernard S, Ducraux A, Nicoulaz AL, Iggo $\mathrm{R}$ (2003) Induction of an interferon response by RNAi vectors in mammalian cells. Nat Genet 34, 263-4.

35. Khan IF, Hirata RK, Wang PR, Li Y, Kho J, Nelson A, et al (2010) Engineering of human pluripotent stem cells by AAV-mediated gene targeting. Mol Ther 18, 1192-9. 